\title{
Temporal Trends of Legacy and Emerging Persistent Organic Pollutants in Inuit Preschoolers from Nunavik (Québec, Canada)
}

\author{
Huguette Turgeon O'Brien', Doris Gagné ${ }^{2}$, Rosanne Blanchet ${ }^{3}$, Julie Lauzière ${ }^{4}$, Carole Vézina ${ }^{5}$, Pierre \\ Ayotte $^{6}$ \\ ${ }^{1}$ School of nutrition, Laval University, 2425, rue de l'Agriculture, Québec City, QC, Canada, G1V 0A6 \\ Huguette.Turgeon-Obrien@fsaa.ulaval.ca \\ ${ }^{2}$ Nutrition DG, 1187, rue du St-Brieux, Québec City, QC, Canada G1Y 2B9 \\ nutritiondg@yahoo.ca \\ ${ }^{3}$ Interdisciplinary School of health sciences, University of Ottawa \\ 35 University Private (THN140), Ottawa, ON, Canada, K1N 6N5 \\ rblan035@uottawa.ca \\ ${ }^{4}$ Department of Family Medicine and Emergency Medicine, University of Sherbrooke \\ 150, place Charles-Le Moyne, Longueuil, QC, Canada J4K 0A8 \\ Julie.Lauziere@USherbrooke.ca \\ ${ }^{5}$ Inuulitsivik Health and Social Services Centre \\ Puvirnituq, QC, Canada J0M 1P0 \\ carole.vezina.csi@ssss.gouv.qc.ca \\ ${ }^{6}$ Department of social and preventive medicine, Laval University and INSPQ \\ 945, avenue Wolfe, Québec City, QC, Canada G1V 5B3 \\ Pierre.Ayotte@inspq.qc.ca
}

\section{Extended Abstract}

Persistent organic pollutants (POPs) can cause adverse effects on ecosystems and human health. Consequently, legacy POPs such as polychlorinated biphenyls (PCBs) and many chlorinated pesticides were banned or severely restricted in the 1970s or early 1980s, whereas most brominated flame retardants (BFRs) and perfluoroalkyl and polyfluoroalkyl substances (PFASs), often referred to as emerging POPs, were phased out in the mid-2000s. To our knowledge, time trends of legacy and emerging POPs have never been determined in Inuit children from Nunavik. In this context, we have assessed temporal variations of POPs in the blood of 245 preschool Inuit children recruited in Nunavik from 2006 through 2010 (mean age: 24.7 months). However, blood contaminant levels were available for 178 to 181 of the participants, due to challenges related to drawing blood from young children. $\mathrm{PCBs}$, chlorinated pesticides, toxaphene and polybrominated diphenyl ethers (PBDEs) were measured in plasma samples by gas chromatography/mass spectrometry (GC/MS). Plasma PFASs [perfluorooctanesulfonate (PFOS), perfluorooctane (PFOA), perfluorohexanesulfonate (PFHxS)] were performed by highperformance liquid chromatography tandem mass spectrometry (HPLC-MS/MS). Analyses were performed only on substances for which $50 \%$ of the samples were above the limit of detection. For brevity, we have grouped the following

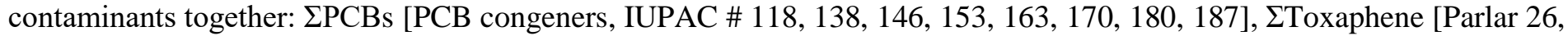
Parlar 50] $\Sigma$ Chlorinated pesticides [DDE, cis-nonachlor, trans-nonachlor, oxychlordane, hexachlorobenzene, $\beta$ hexachlorocyclohexane], इPFASs [PFOS, PFOA, PFHxS] and $\Sigma$ PBDEs [BDE congeners, IUPAC \# 47, 99, 100, 153]. Temporal trends were determined by multiple regression analyses with the year of data collection as the main independent variable. Regression analyses were adjusted for the following confounding variables: age and sex of the child, coast of residence, breastfeeding status, number of smokers in the household and crowding. During the five years of data collection, we found decreasing trends for $\Sigma$ PCBs, $\Sigma$ Chlorinated pesticides and $\Sigma$ Toxaphene $(4.3 \%, 4.2 \%$ and $8.0 \%$ per year respectively), and an increasing trend for $\Sigma$ PFASs (2.6\% per year). However, these trends were not statistically significant. On the contrary, we observed strongly significant decreasing trends for BDE 47 (14.6\% per year, $p=0.0004)$, BDE 99 (13.2\% per year, $p=0.006)$, BDE $100(11.8 \%$ per year, $p=0.006)$, BDE $153(9.2 \%$ per year, $p=0.025)$, and an overall decreasing trend of $13.2 \%$ for $\Sigma$ PBDEs $(p=0.0013)$. The greater reduction observed for BDE 47 and BDE 99 was probably 
due to the fact that these congeners were the major components of the pentaBDE commercial mixture used predominantly in North America and phased out in Canada since 2006. PCBs, chlorinated pesticides and toxaphene continued to decrease, though at a slower rate compared to PBDEs, probably because many of these legacy POPs were banned or severely restricted over three decades ago. Finally, the use of PFOS has been banned only since 2009, thus limiting the possibility of a significant impact on $\Sigma$ PFASs in our study. Continued monitoring of POPs is required in this population, particularly for PBDEs whose levels observed in 2010 were still higher than those reported in many children around the world. 